\title{
Empathy and Compassion as a Caring Personality and Their Functional Neuroimaging in theBrain: A Literature Review
}

\author{
Kuntarti Kuntarti ${ }^{1}$, Yeni Rustina ${ }^{2}$ \\ ${ }^{1}$ Basic Science and Fundamental of Nursing Department, Faculty of Nursing, Universitas Indonesia. \\ Depok, Indonesia, $\bowtie$ kuntarti@ui.ac.id \\ 2 Department of Paediatric Nursing,Faculty of Nursing, Universitas Indonesia, Depok, Indonesia, \\ 凶y_rustina@ui.ac.id
}

\begin{abstract}
The development of caring personalities such as empathy and compassion is crucial for successful therapeutic interaction for human-related professional. The scientific studies have recently revealed functional neural plasticity associated with the training of empathy and compassion. This article reviews the functional neuroimaging of empathy and compassion in the brain. The recent studies have given insights that empathy increased the negative effect and brain activation in the anterior insula and anterior midcingulate cortex brain that associated with empathy for pain and compassion increased the positive effect and brain activation in the ventral striatum, progenual anterior cingulate cortex, and medial orbitofrontal cortex brain. These results indicate that human-related professional such as nurses, psychologists, and other health professionals can feel empathy as pain or distress if these feelings occur continuously as experienced by them. However, the compassion program can reducethis condition. These findings suggest that the cultivation of compassion can be design as a program to maintain empathy and increase resilience and coping strategies in health care, educational setting, and high-stress environment.
\end{abstract}

Keywords:brain, caring personality, compassion, empathy, neural plasticity Padang.

\section{Introduction}

The human-related professional such as nurses, psychologist, medical doctors, and others must have a caring personality. The caring person shows kindness, concern, empathy, altruistic act, compassion, and the other traits included in humanistic behaviors (Adams, 2016; Dewar, 2013; Klimecki, 2017; Klimecki \& Singer, 2015; Pajnkihar, Štiglic, \& Vrbnjak, 2017; Smith, Turkel, \& Wolf, 2012). As a personality trait, there are individual differences that caused by genetic and environmental factors, but Plomin and Nesselroade (1991) in Matthews, Deary, \& Whiteman (2009) suggested that heritability of personality may change over development and the genes affecting a trait need not be the same. The biological bases, namely genes, brain, and hormones determine personality trait as a basic tendency in the Five Factors Theory developed by McCrae and Costa (Feist, Feist, \& Roberts, 2018). Recent, the biological approach to personality has also identified areas 
and pathways within the brain that are associated with the development of personality (Arslan et al., 2017; Carrington \& Bailey, 2009; Fox et al., 2014; Klimecki, Leiberg, Ricard, \& Singer, 2014; Saarela et al., 2007). The functioning of specific brain circuit and activated brain are suited to examining brainenvironment interaction in personality development.

Empathy and compassion are crucial components in the therapeutic interaction of caring relationships. Empathy is our capacity to understand others' feeling that is linked to altruistic behavior (Cunico, Sartori, Marognolli, \& Meneghini, 2012; Tusche, Bockler, Kanske, Trautwein, \& Singer, 2016). However, compassion is a feeling of concern for the suffering of others that is associated with the motivation to help (Klimecki et al., 2014). Compassion is one of the essentials components for individuals to start and maintain relationships of caring (Halldorsdottir, 2012; Kim et al., 2009; Simon-Thomas et al., 2012; Smith et al., 2012), and a key motivator of altruistic behavior (Weng et al., 2013).

The scientific studies have recently revealed functional neural plasticity associated with the training of empathy and compassion. These studies use the neuroimaging of the brain to examine functional neural plasticity by induced training empathy and compassion. This article reviews the literature that reports about the functional neuroimaging of empathy and compassion in the brain. As a result, this review will cover the questions: Which part of the brain is active when someone behaves empathy and compassion? What actions that activate the areas? These results will provide a recommended action to develop a caring personality by the training that activates the emphatic brain and the compassionate brain.

\section{The Empathic Brain}

The most finding of neuroscience studies on neural associated with empathy is empathy for pain perceived in another person (Hein \& Singer, 2008). In the previous studies, Singer et al. (2004) and Singer et al. (2006) investigated an interactive for pain paradigm use functional magnetic resonance imaging (fMRI). In this study, the volunteers in the fMRI scanner receive either pain themselves or perceive pain in another person. The results suggest that parts of the so-called 'pain matrix' - bilateral anterior insula (AI), the rostral anterior cingulate cortex (ACC), brainstem, and cerebellum- were activated when the volunteer experienced pain themselves as well as they saw another person had experienced pain. Thus, both the experience of pain to oneself and the knowledge that the other personis experiencing pain activates the same pain circuits, suggesting a neural stimulation of the suffering of the other person without pain stimulation to our body. Two recent cross-sectional metaanalyses suggest that empathy for pain crucially involves anterior insula (AI) and anterior midcingulate cortex (aMCC) (Lamm \& Singer, 2010, Klimecki et al., 2014).

The other fMRI study on empathy that volunteers viewed pictures or video of painful unknown faces or body parts in the painful situation has revealed a similar pattern of results. Although the neural stimulation of the pain of another person occurs independently of the affective link between the empathizer and the person in pain, the activated brain was similar (Singer et al., 2006). Hein and Singer (2008) also stated that in most fMRI studies, the effect of empathy for pain hadbeen predominately found in AI and ACC. AI and ACC are also involved in general emotional processing and the affective processing of pain in non-empathy conditions. In a previous study by ShamayTsoory, Tomer, Berger, dan Aharon-Peretz (2003) shows that patients with prefrontal lesions, notably when their damage included the ventromedial prefrontal cortex, were significantly impaired in empathy. Their findings suggest that prefrontal structures play an essential part in a network mediating the empathic response and specifically that the right ventromedial cortex has a unique role in integrating cognition and effect to produce the empathic response. However, a study by Saarela et 
al., (2007) also showed that the strengths of activation in the left anterior insula and left inferior frontal gyrus during observation of intensified pain correlated with subjects' self-rated empathy.

\section{The Compassionate Brain}

Many studies about the neural circuit in the brain has an essential role in compassion behavior associated with empathy and almost similar to an emphatic brain. The study conducted by Sareela et al. (2007) showed that someone could encode the intensity estimates for pain in the others' facial expression as a signal that will be interpreted by the brain. This signal would activate AI and ACC. This pain processing in the brain is similar to the emphatic processing when someone sees pain experiences by others person. Viewing faces expressing pain will trigger motor plans that facilitate escape or helping behavior, corresponding to the observed supplementary motor area (SMA) activation. This study demonstrated that the observers' brains react to the intensity differences in pain when observed in the facial expressions of another human being. This reaction will induce emphatic and compassionate feeling of observers. In the cognitive perspective, empathy is the ability to understand the intention, desires, beliefs of another person as resulting from reasoning about the other's state. However, empathy is an affective state that caused by sharing emotions or conscious states of another person like the Sareelaet al. findings.In another study, activation in the midbrain periaqueductal gray (PAG), a region that is activated during pain and the perception of others' pain, induced compassion and that implicated to parental nurturance behaviors. The increased activation in a region near the PAGand the right inferior frontal gyrus (IFG)correlated with self-reports of compassion experience. These results provide preliminary evidence for understanding the neural correlates of important interpersonal dimensions of compassion (caring)(Simon-Thomas et al., 2012).

Different from empathy that only as a feeling, compassion is composed of other-related affective components and a prosocial motivational component. Klimecki, Leiberg, Lamm, and Singer (2012) stated that compassion as rooted feel in a contemplative tradition aimed at cultivating feelings of warmth, love, and care toward a sequence of people in a state of quiet concentration. Using the socioaffective video task (SoVT), the short video sequences based on real-life situations depicting others in distress, and compassion training Klimecky et al., investigated the neural mechanism of compassion by measured functional neural and subjective responses to witnessing the distress of others in SoVT. To reliably assess participants' compassionate responses, theymeasure empathy, positive affect, and negative affect in response to witnessing videos depicting people in distressing situations (high emotion videos, HE) or showing people performing everyday activities (low emotion videos, LE).

Klimecki et al.(2012)findings provide evidence forfunctional neural plasticity induced by compassion training and thus constitute a crucial step in the endeavor of understanding neural plasticity in the affective domain.Participants reported feeling morenegative feeling and empathy toward other people in distressing situations compared with everyday situations. Empathy rating for $\mathrm{HE}$ videos was significantly correlated with total empathy scores $(\mathrm{p}<0.05 ; \mathrm{r}=0.23)$. Compassionate love was correlated with empathy for LE and HE videos ( $\mathrm{p}<0.01, \mathrm{r}=0,29 ; \mathrm{p}<0.001, \mathrm{r}=0.36)$. Neuroimaging results revealed that $\mathrm{HE}$ videos induced activations in a network comprising areas in the occipital, temporal, and parietal lobes, as well as in the subcortical areas including the amygdala.

Conversely, LE elicited stronger activation in the posterior insula, the $\mathrm{mOFC}$, the fusiform, and the precentral gyrus. Results of this study also shown that feelings of compassion consistently increased activations in subcortical structures including the caudate nucleus and striatum, and cortical regions, including the medial orbitofrontal cortex (mOFC) and supplementary motor areas. After the participants received short-term compassion training, the neural effects were consistent with activations in the right $\mathrm{mOFC}$, ventral tegmental area/ substantia nigra (VTA/SN), pallidum, and putamen as well as bilaterally in the precentral gyrus, amygdala, and thalamus and on the left side in 
the pallidum, putamen, middle occipital gyrus, supramarginal, and superior temporal gyrus (Klimecki et al., 2012).

The compassionate brain is associatedwith the loving brain. As Klimecki'shypotheses: given that other-related feeling of warmth and concern are critical components of compassion, they anticipated that loving kindness training would increase activity in the brain regions previously associated with love or affiliation, such as themOFC, the putamen, the middle insula, and the ventral tegmental area/ substantianigra VTA/SN (Klimecki et al., 2012). Previous studies suggested that love, which is one of the strongest compassionate emotions, is known to be associated with brain areas such as the periaqueductal gray (PAG) and the substantianigra (SN)/ventral tegmental area (VTA) (Bartels \&Zeki, 2004). This front-midbrain-ventral striatum/septal region network, is not only implicated in the motivation/reward system but also coincides with brain areas rich in oxytocin/vasopressin receptors that play critical roles in prosocial and social approachbehaviors such as social attachment, maternal care, social cooperation, and so forth (Kim et al., 2009). These findings corroborate the statement that compassion begins fromempathy and love feeling that encourages someone to understand, help, and respond to the suffering of others.

Compassion implies increasing the prosocial motivation to help the other person. The result of Kim's study show that a compassionate attitude towards another's sad expression modulates the activities of the front-midbrain-ventral striatum/septal region network that is known to be implicated in the prosocial/social approach motivation(Kim et al., 2009). Compassion training cultivates a feeling of benevolence and friendliness, strengthening modes of affiliation, and relies on extending caring feelings (Klimecki et al., 2014). Compassion meditation activated brain areas involved withempathy as well as with happy and pleasant feelings (i.e., the left medial prefrontal cortex and the anterior cingulate gyrus) (Engström \& Söderfeldt, 2010), so the emphatic brain related to prosocial motivation and behavior(Hein \& Singer, 2008).

The happy and pleasant feelings in compassion are opposite to pain feeling in the empathy. Empathy training led to an increase of activation in AI and aMCC which is crucial for processing thenegative effect, pain, and cognitive control. The aMCC is part of cingulate that found to be highly connected to dorsal prefrontal regions. Area dorsolateral prefrontal cortex (DPLFC) also be activated in pain processing. However, fMRI on reward processing revealed a more anterior of cingulate cortex activation which converges with the pACC involvement in compassion. This part of the cingulate cortex was shown to be highly connected with ventral striatum and OFC that associated with reward, love, and affiliation (Klimecki et al., 2014).

\section{Conclusion}

The recent studies have given insights that empathy increased the negative effect and brain activation in the anterior insula and anterior midcingulate cortex brain that associated with empathy for pain and compassion increased the positive effect and brain activation in the ventral striatum, progenual anterior cingulate cortex, and medial orbitofrontal cortex brain. This finding provides evidence for functional neural plasticity by compassion training. The compassion training can serve as a new, powerful method for enhancing positive effect in the adverse situation, strengthening personal resources and health, and positive effect to be resilient. Compassion is not only increasing the positive effect on the person who experiences it, but also to the recipients of compassion.

These results indicate that human-related professional such as nurses, psychologists, and other health professionals can feel empathy as pain or distress if these feelings occur continuously as experienced by them. However, the compassion program can reducethis condition. These findings suggest that the cultivation of compassion can be designed as a program to maintain empathy and increase resilience and coping strategies in health care, educational setting, and high-stress environment. 


\section{References}

Adams, L. Y. (2016). The conundrum of caring in nursing. International Journal of Caring Sciences, 9(1), 1-9. Retrieved from www.internationaljournalofcaringsciences.org

Arslan, S., Ktena, S. I., Makropoulos, A., Robinson, E. C., Rueckert, D., \& Parisot, S. (2017). Human brain mapping: A systematic comparison of parcellation methods for the human cerebral cortex. NeuroImage, (April). https://doi.org/10.1016/j.neuroimage.2017.04.014

Bartels, A., \& Zeki, S. (2004). The neural correlates of maternal and romantic love. Neuroimage, 21. 1155-1166.

Carrington, S. J., \& Bailey, A. J. (2009). Are there theory of mind regions in the brain? A review of the neuroimaging literature. Human Brain Mapping, 30(8), 2313-2335.

https://doi.org/10.1002/hbm.20671

Cunico, L., Sartori, R., Marognolli, O., \& Meneghini, A. M. (2012). Developing empathy in nursing students: A cohort longitudinal study.Journal of Clinical Nursing, 21(13-14), 2016-25.

https://doi.org/10.1111/j.1365-2702.2012.04105.x

Dewar, B. (2013). Cultivating compassionate care.Nursing Standard, 27(34), 48-55.

Engström, M., \& Söderfeldt, B. (2010). Brain activation during compassion meditation: A case study. The Journal of Alternative and Complementary Medicine, 16(5), 597-599.

https://doi.org/10.1089/acm.2009.0309

Feist, J., Feist, G. J., \& Roberts, T.-A. (2018). Theories of personality (Ninth edit). New York: McGrawHill Education.

Fox, K. C. R., Nijeboer, S., Dixon, M. L., Floman, J. L., Ellamil, M., Rumak, S. P., ... Christoff, K. (2014). Is meditation associated with altered brain structure? A systematic review and meta-analysis of morphometric neuroimaging in meditation practitioners. Neuroscience and Biobehavioral Reviews, 43, 48-73. https://doi.org/10.1016/j.neubiorev.2014.03.016

Halldorsdottir, S. (2012). Nursing as a compassionate competence: A theory of professional nursing care based on the patient's perspectives. International Journal for Human Caring, 16(2), 7-19.

Hein, G., \& Singer, T. (2008). I feel how you feel but not always: The empathic brain and its modulation. Current Opinion in Neurobiology, 18(2), 153-158.

https://doi.org/10.1016/j.conb.2008.07.012

Kim, J. W., Kim, S. E., Kim, J. J., Jeong, B., Park, C. H., Son, A. R., ... Ki, S. W. (2009). Compassionate attitude towards others' suffering activates the mesolimbic neural system. Neuropsychologia, 47(10), 2073-2081. https://doi.org/10.1016/j.neuropsychologia.2009.03.017

Klimecki, O. (2017). Malleable emotions, (July). https://doi.org/10.13140/RG.2.2.11812.17285

Klimecki, O. M., Leiberg, S., Lamm, C., \& Singer, T. (2012). Functional neural plasticity and associated changes in positive affect after compassion training. Cerebral Cortex, 23(7), 1552-1561. https://doi.org/10.1093/cercor/bhs142

Klimecki, O. M., Leiberg, S., Ricard, M., \& Singer, T. (2014). Differential pattern of functional brain plasticity after compassion and empathy training. Social Cognitive and Affective Neuroscience, 9(6), 873-879. https://doi.org/10.1093/scan/nst060

Klimecki, O. M., \& Singer, T. (2015). Compassion. Brain Mapping: An Encyclopedic Reference, 3, 195-199. https://doi.org/10.1016/B978-0-12-397025-1.00178-0 
Lamm, C., \& Singer, T. (2010). The role of anterior insular cortex in social emotions. Brain Structure and Function, 214, 1-13. https://doi.org/10.1007/s00429-010-0251-3

Matthews, G., Deary, I. J., \& Whiteman, M. C. (2009). Personality traits (Third edit). New York: Cambridge University Press.

Pajnkihar, M., Štiglic, G., \& Vrbnjak, D. (2017). The concept of Watson's carative factors in nursing and their (dis)harmony with patient satisfaction. PeerJ, 5, e2940. https://doi.org/10.7717/peerj.2940

Saarela, M. V., Hlushchuk, Y., Williams, A. C. D. C., Schürmann, M., Kalso, E., \& Hari, R. (2007). The compassionate brain: Humans detect intensity of pain from another's face. Cerebral Cortex, 17(1), 230-237. https://doi.org/10.1093/cercor/bhj141

Shamay-Tsoory, S. G., Tomer, R., Berger, B. D., \& Aharon-Peretz, J. (2003). Characterization of empathy deficits following prefrontal brain damage: The role of the right ventromedial prefrontal cortex. Journal of Cognitive Neuroscience, 15(3), 324-337. https://doi.org/10.1162/089892903321593063

Simon-Thomas, E. R., Godzik, J., Castle, E., Antonenko, O., Ponz, A., Kogan, A., \& Keltner, D. J. (2012). An fmri study of caring vs self-focus during induced compassion and pride. Social Cognitive and Affective Neuroscience, 7(6), 635-648. https://doi.org/10.1093/scan/nsr045

Singer T, Seymour B, O’Doherty J, Kaube H, Dolan R.J., \& Frith C.D.. (2004) Empathy for pain involves the affective but not sensory components of pain. Science, 303:1157-1162.

Singer T, Seymour B, O'Doherty JP, Stephan K.E., Dolan R.J., \& Frith C.D. (2006).Empathic neural responses are modulated by the perceived fairness of others. Nature, 439:466-469.

Smith, M. C., Turkel, M. C., \& Wolf, Z. R. (2012). Caring in nursing classics: An essential resource. Retrieved from https://books.google.com/books?hl=en\&lr=\&id=yTuv-tEuGE0C\&pgis=1

Tusche, A., Bockler, A., Kanske, P., Trautwein, F.-M., \& Singer, T. (2016). Decoding the charitable brain: Empathy, perspective taking, and attention shifts differentially predict altruistic giving. Journal of Neuroscience, 36(17), 4719-4732. https://doi.org/10.1523/JNEUROSCI.3392-15.2016

Weng, H. Y., Fox, A. S., Shackman, A. J., Stodola, D. E., Caldwell, J. Z. K., Olson, M. C., ... Davidson, R. J. (2013). Compassion training alters altruism and neural responses to suffering. Psychological Science, 24(7), 1171-1180. https://doi.org/10.1177/0956797612469537 